\title{
La delincuencia en el discurso de personas en situación de calle de Santiago de Chile
}

\author{
Cinthya Elizabeth Lepin Rueda* \\ Lésmer Antonio Montecino Soto ${ }^{* *}$
}

\section{Resumen}

Este artículo pretende dar cuenta de los recursos argumentativos por medio de los cuales las personas en situación de calle articulan la representación discursiva de la delincuencia vinculada a su identidad. La decisión de profundizar en esta temática desde la perspectiva del Análisis Crítico del Discurso (ACD) se sustenta en que el tópico 'delincuencia' aparece como una categoría recurrente en las entrevistas que conforman el corpus del Proyecto FONDECYT 1071094 "El discurso de las personas en situación de calle de Santiago de Chile".

Palabras clave: Persona en situación de calle - ACD - Representación discursiva - Historias de vida -Delincuencia

\section{Abstract}

This article expects putting into consideration the argumentative resources used for the homeless people as tools for the construction of discursive representation of delinquency linked to their identity. The decision of deepen in this thematic from Critical Discourse Analysis (CDA) perspective it grounds on the fact that the topic 'delinquency' appears like a recurrent category in the interviews of the FONDECYT 1071094 project "The discourse of the homeless people of Santiago, Chile".
\end{abstract}

Key words: homeless people - CDA - discursive representation - Life Stories - Delinquency

* Magíster en Letras, mención lingüística. Pontificia Universidad Católica de Chile. celepin@uc.cl

** Doctor en Filología Hispánica. Universidad de Valladolid. Imontecs@uc.cl 
La delincuencia en el discurso de personas en situación de calle de Santiago de Chile / Cinthya Lepin Rueda, Lésmer Montecino Soto

\section{Introducción}

El presente artículo corresponde a un estudio de caso, de corte cualitativo que sigue los lineamientos de la Grounded Theory (Glaser y Strauss, 1967). En él, analizamos de forma detallada tres historias de vida de personas en situación de calle, cuyo tópico central constituye una de las temáticas más trascendentales para la sociedad chilena, a saber, la delincuencia y la creencia de que las personas en situación de calle son delincuentes. La opinión pública, los medios masivos de comunicación, los índices de Paz Ciudadana, Carabineros de Chile y la Policía de Investigaciones, junto con la voz del Gobierno chileno, ponen de manifiesto que una de las metas del Chile del Bicentenario es la lucha contra la delincuencia. A nivel discursivo, observamos que los actores sociales antes mencionados dan cuenta "del triunfo de la guerra en contra de los antisociales, de los ladrones, de los quebrantadores de la ley, de aquellos seres malignos, anormales y sin valores, que no permiten que la sociedad viva en paz". Al analizar estas calificaciones reproducidas en el discurso cotidiano, nos percatamos de que dicha lucha es en contra de un colectivo abstracto y maligno, lo que ha provocado que olvidemos que se trata de personas que han delinquido. Esta lucha en contra de un colectivo abstracto y maligno ha provocado que olvidemos que se trata de personas, razón por la cual el presente artículo tiene como objetivo dar cuenta de los recursos argumentativos por medio de los cuales las personas en situación de calle articulan su identidad vinculada a la representación discursiva de la delincuencia. Asimismo, pretendemos visibilizar las garantías (topoi) que justifican la representación discursiva de la delincuencia, por un lado, y obtener y describir el paradigma argumentativo por medio del cual se articula la representación discursiva de la delincuencia, por otro.

Este trabajo se inscribe en el desarrollo del Proyecto FONDECYT 1071094 "El discurso de las personas en situación de calle de Santiago de Chile", el cual se adscribe al Análisis Crítico del Discurso (ACD). Para definir a las personas en situación de calle, hemos tomado en cuenta el concepto sociológico elaborado por Red Calle (2008), el cual se establece a partir de tres ejes: la morada, el tiempo y la exclusión. En cuanto al eje I, una persona se encuentra en situación de calle cuando pernocta en lugares públicos o privados, sin contar con una infraestructura que pueda ser denominada como vivienda; carece de alojamiento 
fijo, regular y adecuado, por lo que recurre a hospederías solidarias y comerciales; o bien, participa en programas que ofrecen alojamiento permanente o por periodos importantes con apoyo bio-sicosocial.

En cuanto al segundo eje, las personas en situación de calle pueden ser: ocasionales, recientes, habituales y permanentes. Los ocasionales no suman más de un mes en situación de calle durante el último año; los recientes llevan menos de un mes continuo en dicha situación; los habituales, han permanecido entre un mes y seis meses seguidos o acumulados en el último año en dicha situación; y, por último, los permanentes, han vivido por un período superior a seis meses en situación de calle. Finalmente, el eje III tiene relación con los procesos de exclusión social en los que se ven involucradas estas personas, los que son posibles de visualizar a partir de sub-ejes, tales como la familia, la pareja, el trabajo/escuela, los amigos, la comunidad, la salud y la protección social.

\section{Estado del área}

\subsection{La delincuencia en Chile: los índices criminales}

Para el presente estudio, tomamos en consideración los datos del Anuario de estadísticas criminales (2008), perteneciente a la Fundación Paz Ciudadana, cuya información remite al año 2008. Dichas estadísticas reflejan todos los delitos de mayor connotación social ocurridos durante un año específico. A este respecto, en el año 2007 el total de los aprehendidos de todo el país ascendía a 432.299, número que para el año 2008 aumentó a 477.651. No obstante, las denuncias ocupan un número sumamente mayor y alarmante: en el año 2007 fueron de 1.031.087 y aumentaron a 1.248.412 en el año 2008. Según estas cifras, Carabineros de Chile atiende un total de 9,9 procedimientos por minuto.

\subsection{La situación penitenciaria de Chile}

Por otro lado, la situación de las cárceles en Chile resulta bastante precaria. El hacinamiento de los centros penitenciarios hasta el año 2007 es de un 48\%. La población penal total es de 105.827 personas, de las cuales 53.541 pertenecen al sistema cerrado y 52.286 pertenecen al sistema abierto (medidas alternativas que constan de una sanción que permite continuar desarrollando la vida laboral, familiar y social de la persona: Remisión Condicional de la Pena, Reclusión Nocturna y Libertad Vigilada). Como vemos, existe casi igual número de reclusos en un sistema sin ninguna oportunidad (cerrado) y en otro con medidas que no apuntan directamente a la reinserción social (abierto). Sumado a esto, las cárceles de Valparaíso, Arica y Concepción concentran un poco más del doble de su capacidad.

Con estos datos, en la tasa de reclusión mundial, Chile se ubica en el tercer lugar de América Latina después de Surinam y Guyana y, en el puesto número 
La delincuencia en el discurso de personas en situación de calle de Santiago de Chile / Cinthya Lepin Rueda, Lésmer Montecino Soto

treinta y cuatro a nivel mundial, en el ranking internacional del Centro Internacional de Estudios Penitenciarios de King's College, Universidad de Londres.

No obstante, estos alarmantes antecedentes no parecen importarle a persona alguna. De acuerdo con la opinión pública, "los ladrones, delincuentes o los antisociales" deben 'podrirse' en la cárcel para saldar su deuda con la comunidad que los cobijó. En los massmedia se nos habla de lo preocupante que es la existencia de una 'puerta giratoria' y de la ineficiencia del sistema judicial, situaciones que incrementan la vulnerabilidad que siente la gran mayoría de los chilenos frente a estos verdaderos "enemigos internos".

Dicha sociedad parece no percatarse de que incrementar la tasa de hacinamiento en los complejos penitenciarios no es la mejor solución frente al problema que se reclama, sino que, por el contrario, constituye un agravante más. Las sociólogas Dammert y Díaz (2005a: 5) nos indican que diversas investigaciones dan cuenta de que no hay una correlación entre el incremento de la población carcelaria y la disminución de los delitos denunciados. A su vez, no existen estudios que indiquen de manera evidente que la experiencia carcelaria reduzca la tasa de criminalidad y evite la reincidencia (Dammert y Díaz, 2005b: 6).

\subsection{La postura del Gobierno de Chile}

Debido a esto, a partir del año 2008 se está poniendo en marcha la reforma procesal penal que busca mejorar la efectividad de la experiencia reformadora de la cárcel. No obstante, ninguna de estas leyes y proyectos se relacionan con el mejoramiento de la calidad de vida de la población penal, ni con la reinserción social de la misma o con algún tipo de instrucción formal que estas personas puedan recibir. Tampoco se relacionan con los empleos futuros a los que puedan acceder las personas que cumplen condenas ni con el mejoramiento salarial de los gendarmes. El único proyecto que se relaciona con lo anteriormente expuesto es el que tiene relación con la creación de medidas alternativas. Sin embargo, estas solo hacen que la estancia en la cárcel sea parcial en lugar de ser total y no pretenden directamente optimizar la vida de los reclusos.

Frente a esto, Bruner (2005) sostiene que el gobierno chileno articula el problema delictual a partir de factores económicos por lo que toma en cuenta solo dos aristas: la falta de educación y la criminalidad. De acuerdo con esto, es la falta de una instrucción formal la que lleva a las personas a delinquir, pero, como pudimos percatarnos, no se hace nada por mejorar la educación que reciben quienes cometen delitos. Por el contrario, se creó una ley de responsabilidad adolescente que contribuye con el aumento de los índices de deserción escolar.

Por esto, la mirada hacia el problema de la delincuencia debe ser tridimensional, esto es, debemos tomar en cuenta tanto las conductas delictivas, como el déficit educacional y la desigualdad social. De esta manera, los hechos delictuales podrían explicarse por la falta de educación y por la enorme brecha social 
existente en Chile, la cual genera una carencia importante de oportunidades para los sectores más desposeídos de la sociedad chilena.

\section{Corpus}

Seleccionamos tres historias de vida que resultarán ejemplares con respecto al tópico de la delincuencia. Estas fueron escogidas a partir de las impresiones de las coinvestigadoras y del investigador responsable al momento de ser grabadas. Las tres corresponden al mes de octubre del año 2008. Dos de ellas fueron obtenidas de la Hospedería Padre Lavín (identificadas con las iniciales MS y JM) y una de la Fundación Gente de la Calle (identificada con las iniciales JC). En cuanto a los actores sociales, son todos del sexo masculino y se ubican entre los 25 y los 48 años de edad. Los dos primeros se encuentran en calidad de permanentes (de acuerdo con el eje tiempo propuesto por Red Calle ${ }^{1} 1$ ), ya que llevan 17 y 39 años en situación de calle, mientras que el tercero se encuentra en calidad de reciente, debido a que lleva 9 meses en dicha situación.

\section{Análisis}

\subsection{Las oposiciones discursivas}

La unidad de análisis con la que se trabajó es el episodio temático² (Linell, 1998). Para el presente estudio, los episodios temáticos de delincuencia corresponden a aquellas zonas del discurso en las cuales el actor social en estudio alude a cualquier acto delictual en el que haya participado de forma protagonista o como testigo, ya sea directamente o bien de forma indirecta relevando sub-tópicos que operan como ingreso al episodio temático de delincuencia, por lo que se encuentran supeditados a éste. Por ende, pueden encontrarse de forma dispersa a lo largo de cada discurso.

Dichos episodios se componen de tres partes. Estas son la etapa de instauración (directa o indirecta), la del desarrollo (identificación de las oposiciones discursivas presentes) y la etapa del cierre (identificación del tópico siguiente). Las oposiciones discursivas (Lepin, 2010) corresponden a aquellos ejes semánticodiscursivos compuestos de dos elementos opuestos entre sí. Dichas oposiciones permiten articular las categorías semánticas que focaliza cada emisor en su discurso. En cada episodio, pueden presentarse ambos elementos de la oposición de forma explícita, o bien, puede presentarse uno implícitamente mediante la presencia del elemento contrario.

De esta forma, tenemos la oposición discursiva antes (positivo) / después (negativo) utilizada para dar cuenta de un corte en la vida de los emisores. Cabe

1 Consultar en www.redcalle.cl

2 Topical episodes, en su idioma original. 
La delincuencia en el discurso de personas en situación de calle de Santiago de Chile / Cinthya Lepin Rueda, Lésmer Montecino Soto

destacar que ambos periodos pueden ser negativos o positivos, dependiendo del momento en que se sitúe el actor discursivo.

La segunda oposición discursiva es valores positivos del robo/valores negativos del robo, la cual describe los aspectos de la delincuencia que, axiológicamente, resultan positivos para el actor discursivo, esto es, que le otorguen un progreso social, educacional, económico y valórico, o bien, que resultan negativos para el mismo, es decir, que lo perjudican y producen quiebres sociales, familiares, educacionales.

La tercera oposición discursiva es vida plena / vida con problemas. En esta, se describen de forma más o menos extensa los aspectos que implica tener una vida plena. Cabe destacar que estos aspectos son particulares, por lo que no se sustentan sobre la base de lo positivo o lo negativo. Por el contrario, el actor discursivo manifiesta aspectos que, más allá de ser calificados como buenos o malos, permiten concluir en su conjunto que se tiene una vida feliz y completa, o bien, una vida con obstáculos.

La cuarta oposición discursiva es ladrón antiguo / ladrón moderno, que resulta altamente interesante de analizar debido a que solo aparece en el relato FGC_JC_091008. En esta oposición, el actor discursivo describe extensa y detalladamente las categorías ladrón antiguo (persona 'chora' que roba 'de forma justa' y 'de forma correcta'), y ladrón nuevo (persona que solo es 'chora' a nivel discursivo, que no sabe robar 'de forma justa' y que carece de escrúpulos por lo que no roba de acuerdo con principios que constituyen una ética del delito. Para JC, el ladrón antiguo tenía 'valores', robaba a los más ricos para compartir el monto 'en familia'. Esta familia es el grupo de origen, padres, hermanos y abuelos consanguíneos, y el grupo nuevo, 'la familia de la calle', un clan que se conformó en un espacio abierto, y que actuaban como una verdadera familia, con responsabilidades y roles definidos por las circunstancias.

No obstante, desconocemos la marca temporal que divide la delincuencia en Chile. Solo sabemos que el ladrón antiguo se mantuvo vigente en la época en la que JC robaba y que en el momento de la entrevista (año 2008), está vigente el ladrón nuevo. Por las afirmaciones de JC, es posible inferir que con la aparición masiva de la droga en las calles, las preferencias de las personas que se dedicaban a delinquir comenzaron a cambiar.

La última oposición discursiva es vida en la cárcel / vida fuera de la cárcel. En esta, se describe todo lo que implica vivir dentro de un centro penitenciario: la relación con gendarmes, los efectos del encierro, del hacinamiento, de las divisiones que la población penal hace de sí misma, esto es, entre las personas que violaron, robaron o traficaron, por ejemplo.

Posteriormente, analizamos la estructura argumentativa de la historia de vida en general (Duarte, 1997) y, de forma específica, dimos cuenta de los tipos de datos, los tipos de garantías y los topoi utilizados por los actores discursivos (Toulmin, 2003). Finalmente, para analizar las aserciones o claim (Toulmin, 
2003) observamos si los actores discursivos se representaban de forma agentiva o pasiva al momento de entregar dicha afirmación (Fairclough, 2003).

\section{Resultados}

A partir del análisis realizado concluimos que las tres historias de vida poseen las características de una argumentación retórica (Duarte, 1997: 81) con un alto grado de subjetividad: la recurrencia de los verbos subjetivos tales como sentir, saber, creer y pensar y de los verbos de estado, cuyo exponente principal es el verbo ser. El aspecto que más llama nuestra atención a este respecto es la amplia y especial presencia del verbo juzgar, utilizado de manera implícita a través de la confesión directa y constante que hacen los actores discursivos en sus historias. Los actores discursivos valoran las acciones propias y de los otros, sea como agentes o como beneficiarios. Todos estos rasgos lingüísticos corresponden a un discurso dialógico, que se refuerza con los tópicos subjetivos tratados en las tres historias del corpus y que dan cuenta de las voces internas de cada actor discursivo.

Tomando esto en consideración, concluimos que dichas características son particulares al género discursivo historia de vida que conforma el corpus del proyecto FONDECYT 1071094, debido a que nuestro objetivo principal al momento de tomar cada entrevista consistió en obtener el máximo grado de participación por parte de los entrevistados y minimizar el propio. Por esto, es el actor discursivo el que tiene la mayor parte de los turnos de habla y, en consecuencia, la interacción pierde los rasgos formales de una entrevista semi dirigida y pasa a tener las características de una confesión constituida por relatos apoyados en un fuerte componente descriptivo-narrativo en función argumentativa.

En segundo lugar, nos centramos en los cuatro factores de la argumentación articulados por Toulmin (2003): las garantías, los datos, las aserciones y los respaldos. En cuanto a los datos, observamos que en general en las entrevistas de la hospedería Padre Lavín se presentan datos de primer orden, esto es, aquellos datos que nacen del conocimiento compartido por los interlocutores. Resulta llamativo el uso de este tipo de datos debido a que entrevistador y entrevistado interactúan por primera y única vez. Entonces, la lógica nos indica que al conversar con una persona que no conocemos deberíamos utilizar datos de segundo orden debido a que por medio de estos el hablante recurre a estrategias de convencimiento. No obstante, esto no ocurre así. De esta forma, confirmamos que los actores discursivos articulan sus historias de vida a partir de supuestos que asumen son compartidos por el entrevistador.

Otro caso muy distinto ocurre en la historia de JC, en la cual, el actor discursivo utiliza en su mayoría datos de segundo orden. Al observar detalladamente el tópico central de esta historia de vida nos percatamos de que su temática es sumamente específica, una materia que el actor discursivo domina a cabalidad 
La delincuencia en el discurso de personas en situación de calle de Santiago de Chile / Cinthya Lepin Rueda, Lésmer Montecino Soto

y que, por ende, debe explicar con argumentos sólidos al entrevistador. Si bien en las otras dos historias de vida se utilizan temáticas particulares, estas no constituyen categorías sociales específicas tal como lo es la distinción entre ladrón antiguo y ladrón nuevo.

Por otro lado, analizamos detalladamente las garantías utilizadas en las tres historias de vida. Así, observamos que prácticamente en su totalidad los actores discursivos recurren a garantías sustantivas, es decir, aquellas que provienen del saber epistémico compartido por una comunidad. Además, no se aprecia ningún uso de garantías de motivación o de autoridad. Con estos datos, damos cuenta de la fuerte valoración que los actores discursivos otorgan a las representaciones sociales de la comunidad de habla en la que están insertos. De esta manera, justifican sus aserciones única y exclusivamente mediante topoi determinados. Cabe destacar que los actores discursivos argumentan aludiendo a los topoi que reproducen los medios de comunicación de masas y que muestran aspectos negativos de la delincuencia ('delinquir no es cristiano', 'delinquir impide obtener un trabajo'), como a aquellos topoi que ellos hacen circular en su cotidianidad y que reflejan aspectos positivos de la delincuencia ('un ladrón es una persona, por lo tanto, no es malo', 'las personas que roban no pierden sus valores', 'la persona que roba por necesidad no delinque'). En consecuencia, observamos que los actores discursivos organizan su historia en torno a la desmitificación de los aspectos negativos que otorga la comunidad a las personas que delinquen y que se sustentan en el discurso dominante que no discrimina causas y efectos. En otras palabras, no explica la raíz del problema de la delincuencia, solo da cuenta del hecho delictivo.

En tercer lugar, decidimos analizar en profundidad las aserciones (claim) presentes. Para ello, analizamos si el actor discursivo se presentaba de forma agentiva o pasiva al momento de emitir su aserción. A este respecto, se producen casos muy particulares y atingentes a cada historia. En la historia de vida de MS nos percatamos de que se produce una evolución en la representación del actor discursivo: en la primera mitad de la historia de vida observamos un actor en rol pasivo que es 'víctima de las circunstancias'; en la segunda mitad, en cambio, estamos en presencia de un actor en un rol agente que toma las riendas de sus acciones. La evolución está marcada por la confesión de los grandes quiebres de su vida: la muerte de su madre y la de su pequeña hija. Luego de esto, el actor pasa de ser víctima a ser victimario.

Por otro lado, en la historia de vida de JM observamos un constante vaivén entre un actor en rol pasivo y uno en rol agente. Como ya dijimos, esta historia posee en un menor grado las características de un monólogo por lo que se acerca más a la entrevista semi dirigida, razón por la cual no se observan intervenciones extensas del actor discursivo, así como tampoco una descripción profunda de las situaciones de su vida. En las intervenciones presentes, el actor discursivo no se instancia de forma sostenida en uno u otro rol, sino que cambia constantemente. 
Por último, la historia de vida JC presenta un alto grado de agentividad. De las tres historias analizadas, este es la que más se acerca al registro confesional. Sus intervenciones son sumamente extensas y en estas el actor discursivo describe con profundidad lo narrado en forma detallada. De esta manera, el actor se instancia casi exclusivamente desde un rol agente, que plantea con seguridad las bondades de la delincuencia: su encarcelamiento en la adolescencia y en la adultez, las persecuciones, los atracos y las diversas experiencias delictivas son producto de una decisión de él y no producto de las circunstancias. Solo observamos que el actor discursivo se representa de forma pasiva al momento de dar cuenta del quiebre de su vida: pasar de un niño inocente a un niño adulto ('el JC de antes y el JC de ahora').

\section{Conclusiones}

A partir de lo expuesto, concluimos que la representación discursiva de la delincuencia en las tres historias de vida se articula de acuerdo con el paradigma argumentativo de la desmitificación de los delincuentes como un flagelo social. Los actores discursivos parten de la base de que las representaciones sociales que en Chile se tienen sobre la delincuencia y de las personas que delinquen son negativas: la delincuencia es un "enemigo social" y los delincuentes "son antisociales, sin valores, enfermos", en fin, "un peligro para la sociedad", por lo tanto 'su lugar debe ser la cárcel'. Sin embargo, debe quedar en claro que, si bien en la calle hay personas que delinquen, no son exclusivamente personas en situación de calle. En las historias de vida se aprecia una fuerte conciencia del discurso dominante: el delito merece sanción social, sin embargo son las circunstancias de vida fundadas en la exclusión las que conducen a que ocasional o permanentemente una persona atente contra la propiedad privada de otro. Nadie quiere robar por regla de vida. Las motivaciones son diversas; entre ellas sobresalen la desigualdad de oportunidades, la imposibilidad de insertarse en un ámbito laboral, la falta de educación o de preparación para desempeñar determinados oficios, la desconfianza y el miedo de los que 'están dentro' del sistema que construyen una representación discursiva de las personas en situación de calle como una amenaza.

En época de elecciones ${ }^{3}$, quienes aspiran a dirigir nuestra sociedad nos indican majaderamente que 'trabajarán duro contra la delincuencia', 'atacaran el problema de raíz', 'terminarán con la puerta giratoria' de manera tal de incrementar aún más la ya hacinada población penal. Frente a esto, los actores sociales $y$, en nuestro caso, discursivos posicionan sus propios topoi: las personas que delinquen son, justamente, personas y no un flagelo que debería encerrarse e invisibilizarse para su extinción. Cuando se comprenda esto, recién estaremos

3 Cabe destacar que el presente artículo se escribió durante las elecciones presidenciales chilenas 2010, las cuales tuvieron como candidatos a Jorge Arrate, Eduardo Frei Ruiz Tagle, Sebastián Piñera y Marco Henríquez Ominami, en primera vuelta, y a Eduardo Frei Riz-Tagle y a Sebastián Piñera en la segunda vuelta. 
La delincuencia en el discurso de personas en situación de calle de Santiago de Chile / Cinthya Lepin Rueda, Lésmer Montecino Soto

preparados para comenzar a crear políticas que sean realmente efectivas en la superación de este problema social que nos atañe a todos los ciudadanos.

\section{Bibliografía}

Bruner, J. (2005). "Educación y actividad delictiva: evidencia internacional". Revista Paz Ciudadana, 1-5.

Dammert, L. y J. Díaz. (2005a). “Es la cárcel la solución para la delincuencia?”. Revista Observatorio. Vol. 1, mayo, 1-6. Programa de Seguridad y Ciudadanía: FALCSO, Chile.

Dammert, L. y J. Díaz. (2005b). "El costo de encarcelar". Revista Observatorio. Vol. 9, noviembre, 1-6. Programa de Seguridad y Ciudadanía: FALCSO, Chile.

Duarte, P. (1997). "La argumentación en el intercambio conversacional". En R. Marafioti (comp.). Temas de Argumentación. (pp. 73-85). Buenos Aires: Biblios.

Fairclough, N. (2003). Analysing Discourse: Textual Analysis for Social Research. London: Routledge.

Fundación Paz Ciudadana (2009). "Balance de la delincuencia 2008". Disponible en Fundación Paz Ciudadana http://www.pazciudadana.cl/publs. php?show=CAT\&idCat $=8$

Gendarmería de Chile (2009). "Estadísticas de población penal atendida por Gendarmería de Chile". Extraído el 30 de agosto de 2009 de http://www. gendarmeria.cl/interior_estadistica.html

Lepin, C. "El trabajo en el discurso de las personas en situación de calle de Santiago de Chile". En L. Montecino (ed.) Discurso, pobreza y exclusión en América Latina. Santiago de Chile: Editorial Cuarto Propio. pp. 195-210.

Linell, P. (1998). Approaching dialogue: talk, interaction and contexts in dialogical perspectives. Amsterdam: John Benjamins.

Montecino, L. (2008). "Los indigentes y la construcción discursiva de su identidad aquí-ahora", en M.L. Pardo (ed.) El discurso sobre la pobreza en América Latina.. Santiago: Frasis. pp. 99-122.

Red Calle. (2008). Revisado el 29 de septiembre, 2008, de http://www.redcalle.cl/ define_psc.asp

Toulmin, S. (2003). Los usos de la argumentación. Barcelona, Península. 